\title{
Adaptive Resilient Routing via Preorders in SDN
}

\author{
Eman Ramadan, Hesham Mekky, Braulio Dumba, Zhi-Li Zhang \\ University of Minnesota \\ \{eman, hesham, braulio, zhzhang\}@cs.umn.edu
}

\begin{abstract}
In this paper, we propose and advocate a new routing paradigm - dubbed routing via preorders - which circumvents the limitations of conventional path-based routing schemes to effectively take advantage of topological diversity inherent in a network with rich topology for adaptive resilient routing, while at the same time meeting the quality-of-service requirements (e.g., latency) of applications or flows. We show how routing via preorders can be realized in SDN networks using the "match-action" data plane abstraction, with a preliminary implementation and evaluation of it in Mininet.
\end{abstract}

\section{CCS Concepts}

$\bullet$ Networks $\rightarrow$ Network protocol design; Routing protocols; Network design and planning algorithms;

\section{Keywords}

Routing via Preorders; Adaptive Resilient Routing; Network Failures; Software-Defined Networking; OpenFlow

\section{INTRODUCTION}

Adding more switches and links to increase available parallelism in networks (i.e., scale-out) has been an effective way to meet insatiable application demands for bandwidth. This leads to a far richer network topology, where there are often many diverse paths between two end points. This is not only the case in data center networks, but is also occurring in large enterprise and ISP networks. However, with the growing network scale, measured both in terms of the numbers of switches and links connecting them, the probability of failures also becomes higher, as there are more nodes and links that can potentially fail. To meet ever stringent availability, reliability and quality-of-service (QoS) requirements demanded by applications, resilient routing - e.g., via pro-active fast failover mechanisms - is imperative.

Conventional routing schemes are primarily path-based (notable exceptions include [13] based on [5]). As illustrated

Permission to make digital or hard copies of all or part of this work for personal or classroom use is granted without fee provided that copies are not made or distributed for profit or commercial advantage and that copies bear this notice and the full citation on the first page. Copyrights for components of this work owned by others than ACM must be honored. Abstracting with credit is permitted. To copy otherwise, or republish, to post on servers or to redistribute to lists, requires prior specific permission and/or a fee. Request permissions from permissions@ acm.org.

DCC'16, July 25-28, 2016, Chicago, IL, USA

(C) 2016 ACM. ISBN 978-1-4503-4220-9/16/07 . \$ \$15.00

DOI: http://dx.doi.org/10.1145/2955193.2955204 in Section 2, the notion of paths is both rigid and fragile. As such, it unnecessarily complicates the design of resilient routing. In addition, most fast rerouting schemes do not explicitly consider QoS requirements (e.g., latency) of flows during fail-overs, nor limit the impact of rerouted traffic on other flows (see Section 2 for further discussion on related work). With a logically centralized control plane and a more flexible data plane abstraction based on match-action rules, software-defined networking (SDN) allows for more powerful routing methods to be employed for adaptive resilient routing. However, path-based routing schemes are still commonly adopted, where flows are mapped to individual paths in the data plane via match-action rules for fail-over routing $[7,8,22]$. On the other hand, due to the significantly longer latency inherent in SDN controller and switch interactions, built-in data plane resiliency via local fast rerouting is crucial for high network performance $[2,8,10]$.

Going beyond paths, we advocate a novel routing paradigm, dubbed routing via preorders, that supports adaptive resilient routing and also accounts for the QoS requirements of applications or flows. The main ideas of our proposed routing via preorders are the following: consider a network $G=(V, E)$, and a flow $F$ from a source $s$ to a destination $d$, with a latency requirement $\tau_{F}$ under normal operations (and a possibly relaxed latency requirement $\tilde{\tau}_{F} \geq \tau_{F}$ under failures). We first define a preorder on a node set $U_{s \rightarrow d} \subseteq$ $V, s, d \in U_{s \rightarrow d}$, which gives rise to a directed (sub)graph $\Gamma_{s \rightarrow d}=\left(U_{s \rightarrow d}, \Xi_{s \rightarrow d}\right)$, where each edge of $\Xi_{s \rightarrow d}(\subseteq E)$ is oriented either one-way or bi-directional, $\left(\Gamma_{s \rightarrow d}\right.$ is called a preordered graph or $\operatorname{PrOG}$ in short (see Section 3 for precise definitions of preorder and PrOG). For resilient routing, we simply map $F$ to $\Gamma_{s \rightarrow d}$ without pre-specifying any path: packets of $F$ can dynamically traverse any (directed) path in $\Gamma_{s \rightarrow d}$. Compared with conventional path-based routing, routing via preorders possesses built-in resiliency against failures.

By appropriately computing preorders or PrOGs, we argue that routing via preorders for adaptive resilient routing attains the latency-constrained optimal resiliency property ${ }^{1}$ : it is resilient against arbitrary $k$ link or node failures as long as those failures do not partition $\Gamma_{s \rightarrow d}$ that contains all paths meeting the flow latency requirements. Under routing via preorders, failures are handled by dynamically deactivating certain directed edges that are affected by the failures and activating certain backup directed edges if necessary (see Section 3 for details). In other words, resiliency is achieved without the need for dynamic graph recomputation or "on-

\footnotetext{
${ }^{1}$ This generalizes the $k$-resiliency property proposed in [3].
} 


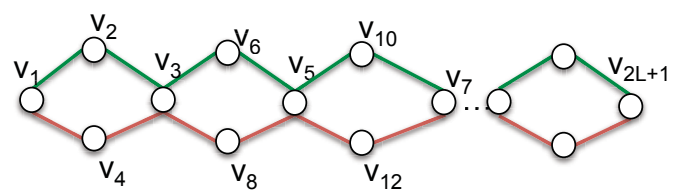

Figure 1: Toy Example

the-fly" graph search to discover available paths as in $[2,13]$, while ensuring the flow latency constraint. Such approach also has the added benefit that it limits the impact of packet rerouting on other flows/applications.

We demonstrate that our proposed routing via preorders can be realized in SDN networks using the match-action data plane abstraction - with small added functionality that switches need to be able to maintain and update certain internal states, and generate an activation tag or a deactivation $\mathrm{tag}$ in response to link/node failure and recovery events. With such capability, we illustrate how routing via preorders can achieve latency-constrained optimal resiliency under arbitrary link/node failures through local data plane operations without involving a centralized SDN controller for route recomputation. Under routing via preorders, we ensure that the SDN controller maintains the full visibility of the data plane as it learns of the network failure or recovery events, since all data plane rules are pre-computed and pre-installed. We have implemented routing via preorders in Mininet with a slightly modified Open vSwitch (OVS). Our initial evaluation results demonstrate the efficacy of routing via preorders in dynamically handling network failures.

\section{LIMITATIONS OF PATH-BASED ROUT- ING AND RELATED WORK}

We first use a simple toy example to illustrate the fundamental limitations of path-based routing. Consider two edge-disjoint paths $P_{1}$ (the upper green path) and $P_{2}$ (the lower red path) from $s=v_{1}$ to $d=v_{2 L+1}$ in Figure 1 , both are of length $2 L$. Let $U$ denote the collection of nodes on $P_{1}$ and $P_{2}$. We see that there are in fact many paths of length $2 L$ (in fact, $2^{L}$ !) from $s$ to $d$ in $U$. To exploit topological diversity, the rigidity of a path as a sequence of nodes and links requires us to enumerate and pre-specify all paths from $s$ to $d$ in $U$ for path-based routing. As the network size increases, the number of paths from $s$ to $d$ can grow exponentially in the worst case - the combinatorial curse $^{2}$. Perhaps more importantly, a path is a very fragile object in that if any link $l=\left(v_{i}, v_{i+1}\right)$ or a node $v_{i}$ along a path fails, the path ceases to exist. For example, using $P_{1}$ as a primary path and $P_{2}$ as a backup path in Figure 1, any two-link failure event with one from $P_{1}$ and the other from $P_{2}$ would render both paths invalid. This is despite the fact that as long as the two failed links are not incident at the same node, there always exists another path (other than $P_{1}$ and $P_{2}$ ) from $s$ to $d$. Only in the case that both links incident on a node $v_{2 k+1}$ fail (or node $v_{2 k+1}$ itself fails), $s$ cannot reach $d$ as the network is partitioned.

Conventional routing schemes are primarily path-based, thus suffer from the fundamental limitations discussed above.

\footnotetext{
${ }^{2}$ When not restricted to shortest paths, in a network with rich topology (i.e., a dense graph where $m=|E| \gg n=|V|$ ) the number of paths can in fact grow in the order of $O(n !)$.
}

Classical IP routing protocols such as RIP and OSPF employ a single shortest-path. In addition, multipath routing has been proposed (see $[14,15,21]$ ), where multiple paths are established between a source-destination pair. Multipath routing can help improve latency and network resilience. However, it is still path-based and thus suffers from the same limitations of path-based routing mentioned above; it also cannot exploit all possible paths between a sourcedestination pair, apart from explicitly enumerating and setting all of them up for multipath routing. While not constrained to shortest paths, MPLS relies on label-switched paths for both primary and protection routing. With IP destination-based forwarding, part of the challenges in designing proactive fast rerouting schemes lies in ensuring consistency between primary and backup paths to avoid forwarding loops under failures. This is achieved in Failure Insensitive Routing (FIR) $[12,17,18]$ by resorting to interfacespecific forwarding (ISF), which provides the first provably correct IP Fast Rerouting algorithm for handling single link (or node) failures. Various IP fast rerouting schemes (see [9, $19,23]$ and references therein) have since been proposed, none of which are resilient against arbitrary multiple link/node failures. As the number of possible (concurrent) link failures increases - thus also the number of possible failure combinations, the combinatorial nature of the problem renders path-based routing approaches less effectual, if not infeasible (see [3] for an interesting negative result regarding the infeasibility of resilient routing under ISF for protecting against arbitrary $k$ link failures when $k>1$ ).

Other alternative resilient routing schemes have also been proposed, e.g., via failure-carrying packets $[11,16]$ where a new path is computed on the fly at each node when encountering failures. The classical link reversal algorithm [5] is the first non-path-based routing scheme, where a new directed acyclic graph (DAG) is constructed in a distributed fashion when encountering a failure, and is provably resilient against arbitrary $k$ link failures in that reachability between any two nodes is guaranteed unless the network is partitioned. Dynamic link reversal mechanisms are adopted in [13] to ensure data plane connectivity under arbitrary link failures. The main drawback of link reversal mechanisms is that in the worst case it takes $O\left(n^{2}\right)$ steps to converge. The authors in [2] cleverly implement a graph search algorithm (e.g., a depth first search) via pre-installed "match-action" rules in SDN switches, and a dynamically adjusted "tag" carried in packets to achieve resiliency against arbitrary link failures. The downside of this scheme is that under failures all packets may take up to $O(n)$ steps in the worst case to reach the destination, as they "walk around" (e.g., in a depth first search manner) the network to search for an available path. Neither [13] nor [2] takes into account the latency constraint when exploring alternative paths. Given that most applications run on TCP, we believe that bounding the latency of packets (both under the normal and failure scenarios) is important, as long delayed packets will likely lead to time-outs, triggering unnecessary packet retransmission; they will further reduce the throughput of applications. Lastly, we would like to point out that various diverse and resilient routing schemes (e.g., $[1,6,20])$ have been "customer-designed" for data center networks, which often only work for specific types of network topologies (e.g., Fat-Tree or Leaf-Spine networks). 


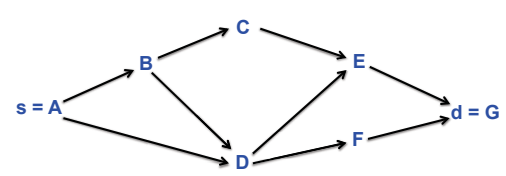

(a) Primary PrOG

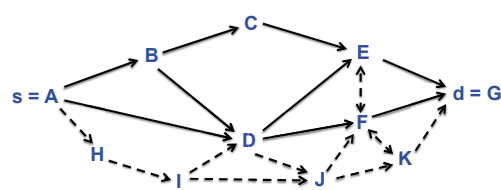

(b) Backup PrOG

Figure 2: Example of Routing Preorders and their associated PrOGs

\section{RESILIENT ROUTING VIA PREORDERS}

Basic Notions and Illustration. Let $G_{s \rightarrow d}=\left(U_{s \rightarrow d}, \Xi_{s \rightarrow d}\right)$ be a connected ${ }^{3}$ subgraph of $G$, where $s, d \in U_{s \rightarrow d} \subseteq V$. We will orient the edges of $G_{s \rightarrow d}$ to create a special preorder on $U$, referred to as a routing preorder $\left(\Gamma_{s \rightarrow d}\right)$ from $s$ to $d$.

Mathematically, a preorder $\lesssim$ on a node set $U$ is a binary relation that is reflective and transitive ${ }^{4}$. For any $u, v \in U$, if $v \lesssim u$, we say $v$ is a predecessor of $u$, and $u$ a successor of $v$; in addition, $v$ is a child of $u$, and $u$ a parent of $v$, if $v \lesssim u$ but $u \not ू v$ (we denote this relation by $v \lesssim u$ ), and $\nexists w \in U$ such that $v \lesssim w \lesssim u$. Given $S \subseteq U, w \in U$ is called an upper bound of $S$, if $\forall v \in S, v \lesssim w$, and it is a least upper bound of $S$, if for any upper bound $w^{\prime}$ of $S, w \lesssim w^{\prime}$. The (greatest) lower bounds of $S$ can be similarly defined. We say a preorder is a (routing) preorder from $s$ to $d$ (and $U_{s \rightarrow d}$ a routing cover), if the following conditions are met: i) $s$ is the unique greatest lower bound of $U$, and $d$ is the unique least upper bound of $U$; and ii) for any $u \in U, s \lesssim u \lesssim d$.

Figure 2 shows an example of a routing preorder from $s$ to $d$, where $v \rightarrow u$ indicates $v$ is a child of $u$, i.e., $v \lesssim u$; and $v \leftrightarrow u$ indicates $u \lesssim v$ and $v \lesssim u$ (in this case we refer to $u$ and $v$ as siblings). Intuitively, the condition ii) above implies that, for any $u \in U$, there is a directed path (or a chain) from $s$ to $u$ and from $u$ to $d$. We call the directed graph induced by a (routing) preorder as a preordered graph or $\operatorname{PrOG}$ in short. As bi-directed edges are allowed in $\operatorname{PrOG}$, it is in general not a directed acyclic graph (DAG).

Given a flow $F$ from $s$ to $d$, instead of mapping it to a single path or multiple paths for routing, we select a routing cover $U_{s \rightarrow d}$ and construct a preorder $\lesssim_{s \rightarrow d}$ for routing packets of $F$ from $s$ to $d$ : at any node $u(\neq d) \in U_{s \rightarrow d}$, packets of $F$ can be proportionally routed to either one of its parents and may also to one of its siblings. In other words, packets are dynamically routed along all feasible paths contained in the PrOG by appropriately merging and splitting traffic at each node, thus utilizing all relevant links, without the need to enumerate and specify all paths. With an appropriately chosen PrOG, routing via preorders has built-in resiliency. Consider again the toy example in Figure 1 where the routing PrOG from $s=v_{1}$ to $d=v_{2 L+1}$ is defined by orienting all edges from left to right. In contrast to path-based routing (say, with two equal-cost, edge-disjoint paths), routing via preorders, using this PrOG, is resilient against arbitrary link failures as long as they do not partition the network. In fact, we argue that it is possible to construct PrOGs in such

\footnotetext{
${ }^{3}$ Namely, any node in $U_{s \rightarrow d}$ can reach any other node in $U_{s \rightarrow d}$ without traversing any node outside $U_{s \rightarrow d}$. In particular, $G_{s \rightarrow d}$ is $s-d$ connected. For conciseness, we drop $s \rightarrow d$ from $U_{s \rightarrow d}$ when the context is clear.

${ }^{4}$ We note that if $\lesssim$ is antisymmetric, it yields a partial order, denoted by $\prec$ on $U$. $(U, \lesssim)$ is called a preordered set or proset, and $(U, \prec)$ is a partially ordered set or poset, yielding a DAG.
}

a manner that routing via preorders attains the (latencyconstrained) optimal resiliency as we will expound on further below.

Preorder Selection for Optimal Resilient Routing. Clearly, what preorder (or PrOG) is selected for routing a flow determines its resiliency. In general, with more nodes and edges included in the routing PrOG, the added diversity increases the built-in resiliency against link or node failures. However, the enhanced resiliency is achieved at the expenses of increasing latency and latency variability of the PrOG. Bounding the overall latency and latency variability under both normal operations and under failures is important for delay-sensitive (e.g., interactive) applications. It can also affect throughput-sensitive applications (e.g., bulk transfer), as re-ordered packets can trigger TCP time-out and unnecessary duplicate packet transmission, thus reducing the overall application throughput. With bounded latency and latency variability, we can also effectively employ GRO and other mechanisms [6] at the destination to put out-of-ordered packets in order before passing them on to TCP.

Consider a network $G=(V, E)$ with a link latency matrix $\Phi=\left[\phi_{i j}\right]$, where $\phi_{i j}$ indicates the latency of link $(i, j)$, if $(i, j) \in E$; otherwise $\phi_{i j}=0$. The latency of a path $P$ in $G$, $\phi(P)$, is thus the sum of its links. For simplicity of exposition, we will equate path length with its latency. Consider a flow $F$ from $s$ to $d$ with a latency requirement $\tau_{F}$ under normal operations (and a possibly relaxed latency requirement $\tilde{\tau}_{F} \geq \tau_{F}$ under failures). We would like to construct a primary $\tau_{F}$-complete routing preorder, or equivalently a PrOG, $\Gamma_{s \rightarrow d}\left(\tau_{F}\right)$, such that all paths in $\Gamma_{s \rightarrow d}\left(\tau_{F}\right)$ meet the criterion, $\phi(P) \leq \tau_{F}$, and also construct a backup $\tilde{\tau}_{F}$-complete PrOG $\Gamma_{s \rightarrow d}\left(\tilde{\tau}_{F}\right)$, such that $\Gamma_{s \rightarrow d}\left(\tau_{F}\right) \subseteq \Gamma_{s \rightarrow d}\left(\tilde{\tau}_{F}\right)$. This leads us to introduce the following notion: a $\operatorname{PrOG} \Gamma\left(\tau_{k}\right)$ is said to be $\tau_{k}$-complete if any path $P$ from $s$ to $d$ in $G$ such that $\phi(P) \leq \tau_{k}$ is contained in $\Gamma\left(\tau_{k}\right)$ (as a directed path). A $\tau_{k}$-Complete PrOG can be constructed using a two-phase process based on a modified version of breadth-first search that we sketch below: i) we start with the destination $d$ and perform a breadth first search, where at each step we record for each node, its minimum latency, and the minimum latency of its neighbors; ii) then, we start with the source $s$, retrace the steps and prune any branches whose latency exceeds $\tau_{k}$. The complexity of this algorithm is $O\left(n^{2}\right)$. Due to space limitation, the detailed description of the algorithm and its correctness proof are omitted here.

As an illustration, consider the simple network shown in Figure 3a where link weight indicates latency. Figures 3b-d show three $\tau$-complete PrOGs for $s=1$ and $d=6$, with $\tau_{1}=3, \tau_{2}=5$, and $\tau_{3}=15$. Given a flow $F$ from $s$ to $d$ with a latency $\tau_{F}$, we can simply pick one of $\tau_{k}$ 's, e.g., the largest one, such that $\tau_{k} \leq \tau_{F}$. Routing via preorders using a $\tau$-complete $\operatorname{PrOG} \Gamma_{s \rightarrow d}(\tau)$ has the following latency- 


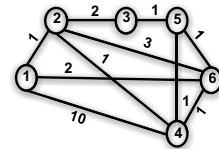

(a) Network

Topology

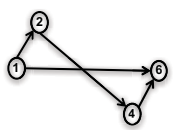

(b) $\tau_{1}=3$

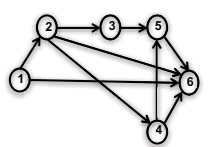

(c) $\tau_{2}=5$

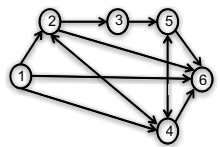

(d) $\tau_{3}=15$

Figure 3: $\tau_{k}$-complete routing preorders

constrained optimal resiliency property: it is resilient against arbitrary $k$ link or node failures as long as those failures do not partition $\Gamma_{s \rightarrow d}(\tau)$ while still meeting the latency requirement $\tau$. This property follows from $\tau$-completeness of the PrOG used for routing as well as the monotonicity of $\tau$ complete PrOGs under failures: let $\hat{G}$ be the network under $k$ link (or node) failures; clearly $\hat{G} \subset G$. If $\hat{\Gamma}_{s \rightarrow d}(\tau)$ is the $\tau$-complete PrOG for $\hat{G}$, then $\hat{\Gamma}_{s \rightarrow d}(\tau) \subseteq \Gamma_{s \rightarrow d}(\tau)$. Hence if $k$ failures partition $\Gamma_{s \rightarrow d}(\tau)$, but $s$ can still reach $d$ after these failures, namely, there is a path $\tilde{P}$ from $s$ to $d$ in $\hat{G}$, then $\phi(\tilde{P})<\tau$.

Resilient Routing via Preoders: Handling Failures and Recoveries. For resilient routing via preorders, we employ a $\tau_{F}$-complete $\operatorname{PrOG}^{5} \Gamma\left(\tau_{F}\right)$ as the primary preorder for routing under normal operations with the latency requirement $\tau_{F}$, and a $\tilde{\tau}_{F}$-complete $\operatorname{PrOG} \Gamma\left(\tilde{\tau}_{F}\right)$ where $\tilde{\tau}_{F} \geq \tau_{F}$ as the backup preorder for resilient routing under failures. As $\Gamma\left(\tau_{F}\right)$ is contained in $\Gamma\left(\tilde{\tau}_{F}\right)$ i.e., $\Gamma\left(\tau_{F}\right) \subseteq \Gamma\left(\tilde{\tau}_{F}\right)$, the directed edges in $\Gamma\left(\tau_{F}\right)$ are considered primary links which are used to forward packets of $F$ during normal operations, whereas the directed edges in $\Gamma\left(\tilde{\tau}_{F}\right) \backslash \Gamma\left(\tau_{F}\right)$ are considered backup edges which may only be invoked for packet forwarding during failures. These backup nodes/edges are installed in the relevant switches as (lower priority) backup rules and get invoked when there are failures in the network. Hence, resilient routing with $\tilde{\tau}_{F}$-resiliency is achieved under arbitrary link/node failures by dynamically deactivating certain directed edges that are affected by the failures and activating certain backup directed edges when necessary. In the following, we will describe how failures and recoveries are handled via the deactivation and activation processes, using the PrOG in Figure 2a as an example.

Deactivation Process: Suppose the link $F \rightarrow G$ goes down. This failure renders $F$ a sink (i.e., it has no outgoing link). In this case, $F$ simply deactivates the incoming link $D \rightarrow F$ by notifying $D$ not to forward packets from $s=A$ to $d=G$ to it. However, this does not affect the reachability from $A$ to $G, D$ simply uses the other outgoing link $D \rightarrow E$ to forward packets from $A$ to $G$. Suppose shortly afterwards, link $D \rightarrow$ $E$ goes down, which renders $D$ a sink. This would trigger $D$ to deactivate its two incoming links, $A \rightarrow D$ and $B \rightarrow D$, and notify its two neighbors. Any existing packets destined to $d=G$, that are buffered at $D$, will simply be rerouted back to $A$ or $B$, if they have not exceeded their latency deadline. As a result of these failures and subsequent (local) actions at the affected nodes, the original PrOG dynamically shrinks by shedding the deactivated links, and the packets from $s=A$ are now routed solely in the remaining unaffected portion of the original PrOG, namely a sub-PrOG on the node set $\{A, B, C, E, G\}$.

Activation Process: When a failed link or node comes back up, a recovery process is initiated to re-activate the relevant

\footnotetext{
${ }^{5}$ For conciseness, we drop $s \rightarrow d$ from $\Gamma_{s \rightarrow d}(\tau)$ when the
} context is clear. portion of the PrOG. Suppose link $F \rightarrow G$ comes back up, then node $F$ is not a sink anymore, which triggers it to activate its incoming link $D \rightarrow F$ by notifying $D$ to forward packets from $A$ to $G$ to it. Thus, node $D$ is not a sink anymore as well, which triggers it to activate its incoming links $A \rightarrow D$ and $B \rightarrow D$ by notifying them. This allows packets from $A$ to $G$ to be routed through the shortest path again (assuming all links have the same weight), i.e., the path $A \rightarrow D \rightarrow F \rightarrow G$. Hence, we emphasize that another key advantage of our routing via preorders is that when failed links/nodes are all recovered, it is guaranteed to return to the same PrOG that was originally selected for resilient routing.

Compared to existing resilient schemes such as $[2,13]$, which also guarantee resiliency against arbitrary link failures, our proposed routing via preorders does not require any dynamic DAG recomputation (which in the worst case takes $O\left(n^{2}\right)$ steps to converge), or on-the-fly graph search by re-routing packets along various paths to search for an available path (which in the worse case takes $O(n)$ steps), our scheme is far simpler and only involves deactivating certain pre-installed rules (directed edges) when a node becomes a sink. Neither [13] nor [2] can ensure only paths satisfying certain latency constraints are used for forwarding under failures. Furthermore, only a small portion of packets, that have already been forwarded to the deactivated portion of the PrOG, need to be rerouted, if they have not exceed their latency deadlines; otherwise they will be dropped. This is in contrast to [13] where during the convergence period all packets will be subjected to bouncing around in the network until a new DAG is found, and to [2] where after failures all packets will "walk around" (with repetitive returns to the sources) in the network following a fixed procedure dictated by the graph search algorithm implemented via SDN rules.

\section{REALIZATION IN SDN}

In this section, we show that routing via preorders can be realized in SDN, and how packet forwarding is performed under both normal and failure scenarios. We will also briefly touch on the issues and challenges involved - as routing via preorders also requires some additional functionality that goes beyond existing OpenFlow switches.

The controller computes the routing preorder that satisfies the latency constraints for each flow, then installs the corresponding rules in the relevant switches. Each switch forwards packets using the match-action data plane abstraction, with small added functionality that switches ${ }^{6}$ need to be able to maintain and update certain internal states, and generate an activation tag or a deactivation tag that can be piggybacked in data packets in response to link/node failure and recovery events. Additionally, the switch performs some actions to handle link activation, deactivation and failure.

\footnotetext{
${ }^{6}$ Switch and node are used interchangeably.
} 
Under conventional path-based routing, a flow $F$ is first mapped to a pre-selected path $P$, then the SDN controller sets up the corresponding flow entries in the switches along the path. In routing via preorders, we map a flow $F$ to a primary PrOG and a backup PrOG, which are translated into a set of match-action rules to be installed in the relevant switches. Preorders can be realized in SDN as match-action rules by using group tables introduced in OpenFlow 1.1.0 [4]. The ingress port (in-port) will always be part of the match fields, so as to ensure only packets from incoming links that are part of the primary or backup PrOGs will be forwarded to an eligible outgoing link.

Match-Action Rules, Switch States, and Packet Forwarding. Using the PrOGs in Figure 2a (primary) and Figure $2 \mathrm{~b}$ (backup) as an example. Consider node $D$, the match-action rules for which are shown in Figure 4. At each switch, we need to differentiate between primary and backup links. This can be realized using a "fast failover" group table entry. When a packet of flow $F$ destined to $d=G$ arrives at node $D$, it is matched, along with its ingress port, against the rules associated with the flow $F$. Since there are two active primary outgoing links $D \rightarrow E$ and $D \rightarrow F$ (thus the rules associated with them are inserted in a separate group (GrpId:200), which has higher priority than that with the backup outgoing link $D \rightarrow J$ (GrpId:300). The is because "fast failover" group type in OpenFlow executes the first live bucket, thus the order matters, and the liveness of each bucket is controlled by the liveness of its associated port or group. Hence, the primary and backup outgoing links are listed into different buckets in different groups, with the group of primary links listed first in the "fast failover" group (GrpId:100). Only, when all the primary outgoing links are not live i.e., (GrpId:200) is inactive, then the backup links are used to forward packets. Later on, if the backup links also become inactive i.e., (GrpId:300) is inactive, the switch forwards packets back on any of its in_ports, and invokes the "deactivation process" described earlier (GrpId:400).

Since not all paths in the primary $\operatorname{PrOG} \Gamma\left(\tau_{F}\right)$ meet the latency requirement $\tau_{F}$ under normal operations, nor in the backup PrOG $\Gamma\left(\tilde{\tau}_{F}\right)$ meet the (relaxed) latency requirement $\tilde{\tau}_{F}$ under failures, only a subset of outgoing links at each node $i$ may be eligible for forwarding packets under normal operations and failures. This can be accomplished via two steps. First, we insert two header fields in packets representing its latency requirements, a latency field (similar to the standard TTL field) and a latency offset field. At the source $s$, the latency field is set to $\tau_{F}$ and the latency offset field is set to $\tilde{\tau}_{F}-\tau_{F}$. Any time a packet is forwarded along a link $i \rightarrow j$, the latency field is decremented by $\phi_{i j}$; the packet is dropped when this value reaches 0 . In the following, we will describe how the latency offset field is used when network failures are encountered. Second, for each outgoing link $i \rightarrow j$, we maintain a (per-destination) latency state information which records the minimal (path) latency achievable from node $i$ when forwarding a packet along the outgoing link $j$ to destination $d$, denoted by $\tau^{d}(i \rightarrow j)$. Hence, given a packet with its current latency header value $\tau$, only those outgoing links with $\tau^{d}(i \rightarrow j) \leq \tau$ are eligible for forwarding the packet.

To handle failure (and recovery) events, the state of each outgoing link $i \rightarrow j$ indicates whether the match-action rule associated with the (primary or backup) outgoing link $i \rightarrow j$ is active or inactive. For example, if the outgoing port $j$ is

\begin{tabular}{|c|c|c|c|c|c|}
\hline \multicolumn{2}{|c|}{ Flow Table } & \multicolumn{4}{|c|}{ Group Table } \\
\hline Match & Action & \multirow{2}{*}{$\begin{array}{c}\text { Group } \\
\text { Id }\end{array}$} & \multirow{2}{*}{$\begin{array}{l}\text { Group } \\
\text { Type }\end{array}$} & \multirow{2}{*}{$\begin{array}{l}\text { Action } \\
\text { Bucket }\end{array}$} & \multirow[t]{2}{*}{ State } \\
\hline $\mathrm{src}=\mathrm{A}, \mathrm{dst}=\mathrm{G}$, in_port $=\mathrm{A}$ & Group: 100 & & & & \\
\hline src $=A$, dst $=G$, in_port $=B$ & Group: 100 & \multirow{2}{*}{100} & \multirow{2}{*}{$\begin{array}{c}\text { fast } \\
\text { failover }\end{array}$} & Group: 200 & Active \\
\hline \multirow{2}{*}{$\mathrm{src}=\mathrm{A}, \mathrm{dst}=\mathrm{G}$, in_port $=\mathrm{E}$} & \multirow{2}{*}{$\begin{array}{l}\text { - Invoke deactivation } \\
\text { - Group: } 100\end{array}$} & & & $\begin{array}{l}\text { Group: } 300 \\
\text { Group } 400\end{array}$ & $\begin{array}{l}\text { Acive } \\
\text { Active }\end{array}$ \\
\hline & & \multirow{2}{*}{200} & \multirow{2}{*}{ select } & Output: F & Active \\
\hline \multirow{2}{*}{ src $=A$, dst $=G$, in_port $=F$} & \multirow{2}{*}{$\begin{array}{c}\text { - Invoke deactivation } \\
\text { - Group: } 100\end{array}$} & & & Output: $E$ & Active \\
\hline & & 300 & select & Output: J & Active \\
\hline Match & Action & \multirow{3}{*}{400} & \multirow{3}{*}{ select } & \multirow{3}{*}{\begin{tabular}{|c|} 
- Output: \\
in_port \\
- Invoke \\
deactivation \\
\end{tabular}} & \multirow[t]{3}{*}{ Active } \\
\hline Activation tag [src, dst, in_port] & Invoke activation & & & & \\
\hline Deactivation tag [src, dst, in_port] & Invoke deactivation & & & & \\
\hline
\end{tabular}

Figure 4: Match-action rules for switch $D$

down or when a deactivation notification is received from node $j$ (see below), the corresponding rule is marked inactive. Hence at node $i$, if it detects a failure event, e.g., an outgoing link/port is down (or a neighboring node is unresponsive), it would mark the rules associated with the outgoing link/port as inactive. If the link/port/node is recovered, the rules are reset to active. The switch also keeps track of the total number of active outgoing links/ports associated with the ruleset of flow $F$. If this number becomes 0 , this renders switch $i$ a sink, namely, no active outgoing link is available for forwarding packets of $F$. This triggers switch $i$ to invoke the deactivation process: it will generate a special deactivation notification containing the appropriate information (e.g., the source-destination pair associated with the affected PrOG) and send it back along all its (active) incoming links $h \rightarrow i$; upon receiving this deactivation notification, the downstream switch $h$ would mark the outgoing link $h \rightarrow i$ inactive. If this results in switch $h$ becoming a sink, it will continue this deactivation process further downstream. The deactivation notification can be implemented either as a tag inserted in the packet header and can be piggybacked by any data packet traveling along the (reverse) link $i \rightarrow h$; or it can be implemented as a special error message sent by $i$ to $h$. Either mechanisms can be realized as a match-action rule, as shown in Figure 4. When a failed link or node comes back up, a recovery process can be initiated to re-activate the relevant portion of the PrOG.

Packet Forwarding Under Failures. In the event of network failures, e.g., when both the primary outgoing links $D \rightarrow E$ and $D \rightarrow F$ become inactive, the group associated with them also becomes inactive, then the eligible backup outgoing link $i \rightarrow j^{\prime}$ can be invoked for packet forwarding. When a packet is sent along an eligible backup link $j^{\prime}$ (here, $j^{\prime}=J$ ), we first add the value in the latency offset field to the latency header field before the latency header is decremented by $\phi_{i j^{\prime}}$ and reset the latency offset to 0 . If the updated value in the latency header field $>0$, the packet is forwarded; otherwise it is dropped. This way we ensure that the packet will meet its (relaxed) latency requirement.

We have implemented routing via preorders in Mininet with a slightly modified Open vSwitch (OVS) using version (2.4) which supports group tables. We added modules to introduce adding extra parameters to group buckets, manipulate extra header fields in packets, dynamically update the state of the ports (logically and not only physically $(\mathrm{Up} /$ Down $)$ ), select eligible outgoing links, support the activation \& deactivation of links, and exchange activation \& deactivation tags. We also used a patch to support OpenFlow group chaining as it was not supported before ver- 


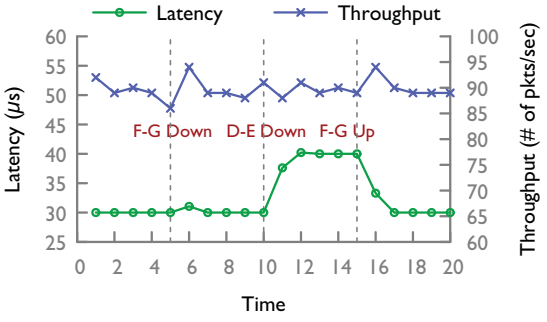

Figure 5: Latency \& Throughput

sion (2.5). As part of our proposed routing paradigm, we will investigate different methods for efficiently representing and compacting the match-action rules and state information in a way to minimize the space requirements.

\section{PRELIMINARY EVALUATION}

We evaluated routing via preorders in Mininet using the topology shown in Figure 2a. A UDP traffic generator at host $h_{1}$, attached to node $A$, sends packets to host $h_{2}$, attached to node $G$, with a latency requirement $\tau_{F}=40 \mu \mathrm{s}$ under normal operations, and a relaxed latency $\tilde{\tau}_{F}=60 \mu \mathrm{s}$ under failures. For each link $l=(i, j) \in E$, the value of $\phi(i, j)=10 \mu s$ determines the latency of routing packets along the link $l$. The flow entries in each switch are prioritized to prefer shorter paths, and the neighbor id is used as a tie-breaker, i.e., in Figure 2a the default path is $A \rightarrow D \rightarrow F \rightarrow G$.

The traffic lasts for 20 seconds, sending around 90 packets per second. In normal operations, packets traverse the shortest path. For link failures, we follow the same scenario described earlier in Section 3. After 5 seconds, we fail the link $F \rightarrow G$, then $F$ becomes a sink node, and $D$ uses its other outgoing link $E$. Thereafter, packets traverse the path $A \rightarrow D \rightarrow E \rightarrow G$, which has the same latency as the previous path. Transient packets at node $F$ are rerouted back to $D$, which forwards them to $G$ through $E$ meeting their relaxed latency $\tilde{\tau}_{F}$. After another 5 seconds, we fail the link $D \rightarrow E$, which makes $D$ a sink node. Thus, $D$ deactivates its incoming links $A \rightarrow D$ and $B \rightarrow D$. Now, the only remaining path is $A \rightarrow B \rightarrow C \rightarrow E \rightarrow G$. Finally, after another 5 seconds, the link $F \rightarrow G$ is brought up, and triggers $F$ to activate its incoming link $D \rightarrow F$. Then, $D$ activates its incoming links $A \rightarrow D$ and $B \rightarrow D$. Hence, $A$ sends packets to $G$ through the default shortest path again $A \rightarrow D \rightarrow F \rightarrow G$.

Figure 5 shows the average latency of the packets received at $G$ based on the links they traversed. We see that the latency reflects using the paths in the order explained above. After the failure of the link $F \rightarrow G$, the latency increased slightly for a short time due to rerouting the transient packets. Moreover, our proof-of-concept prototype shows that the connectivity between the two hosts is not affected by link failures as long as there is a path connecting them, and the throughput is not affected as shown in Figure 5. Furthermore, packets were transmitted within the specified (relaxed) latency constraint of the flow, and there was no packet loss. This is due to the deactivation mechanism which trims edges leading to sink nodes, while rerouting transient traffic to another outgoing port which can reach the destination, without the need to change routing rules or involve the controller.

\section{CONCLUSION}

In this paper, we have proposed and advocated a new routing paradigm - routing via preorders - which circumvents the limitations of conventional path-based routing schemes. We illustrated that routing via preorders can effectively take advantage of topological diversity inherent in a network with rich topology for adaptive resilient routing, while at the same time meeting the quality-of-service requirements (e.g., latency) of applications or flows. We have discussed how routing via preorders can be constructed to meet latencyconstrained optimal resiliency, and showed how routing via preorders can be realized in SDN networks using the "matchaction" data plane abstraction. Finally, we presented a proofof-concept prototype highlighting the advantages of our proposed routing paradigm in Open vSwitch.

\section{ACKNOWLEDGMENT}

We are grateful to our shepherd Robert Ricci and the reviewers for their comments and suggestions. This research was supported in part by DTRA grant HDTRA1-14-1-0040, NSF grants CRI-1305237 and CNS-1411636 and a Raytheon/NSF subcontract $9500012169 / \mathrm{CNS}-1346688$.

\section{REFERENCES}

1] M. Alizadeh, et al. Conga: Distributed congestion-aware load balancing for datacenters. In SIGCOMM, 2014.

[2] M. Borokhovich, et al. Provable data plane connectivity with local fast failover: Introducing openflow graph algorithms. In HotSDN, 2014.

[3] J. Feigenbaum, et al. On the resilience of routing tables. arXiv preprint, 2012.

[4] O. N. Foundation. OpenFlow Switch Specification, version v1.1.0, February 28, 2011.

[5] E. M. Gafni et al. Distributed algorithms for generating loop-free routes in networks with frequently changing topology. IEEE Transactions on Communications, 1981.

[6] K. He, et al. Presto: Edge-based load balancing for fast datacenter networks. In SIGCOMM, 2015.

[7] C.-Y. Hong, et al. Achieving high utilization with software-driven wan. In SIGCOMM, 2013.

[8] H. Kim, et al. Coronet: Fault tolerance for software defined networks. In ICNP, 2012.

[9] A. Kvalbein, et al. Fast ip network recovery using multiple routing configurations. In INFOCOM, 2006

[10] K.-W. Kwong, et al. On the feasibility and efficacy of protection routing in IP networks. In INFOCOM, 2010.

[11] K. Lakshminarayanan, et al. Achieving convergence-free routing using failure-carrying packets. ACM SIGCOMM CCR, 2007.

[12] S. Lee, et al. Proactive vs reactive approaches to failure resilient routing. In INFOCOM, 2004.

[13] J. Liu, et al. Ensuring connectivity via data plane mechanisms. In $N S D I, 2013$.

[14] M. K. Marina et al. On-demand multipath distance vector routing in ad hoc networks. In Network Protocols, 2001.

[15] M. K. Marina et al. Routing in mobile ad hoc networks. In Ad Hoc Networks. 2005.

[16] S. Nelakuditi, et al. Blacklist-Aided Forwarding in Static Multihop Wireless Networks. In SECON, 2005.

[17] S. Nelakuditi, et al. Fast local rerouting for handling transient link failures. Transactions on Networking, 2007.

[18] S. Nelakuditi, et al. Failure insensitive routing for ensuring service availability. In $I W Q o S, 2003$.

[19] P. Pan, et al. Fast reroute extensions to rsvp-te for lsp tunnels, 2005.

[20] A. Singla, et al. Jellyfish: Networking data centers randomly. In NSDI, 2012

[21] J. Tsai et al. A review of multipath routing protocols: From wireless ad hoc to mesh networks. In $A C o R N, 2006$.

[22] R. Wang, et al. Openflow-based server load balancing gone wild. In Hot-ICE, 2011.

[23] X. Yang, et al. Nira: a new inter-domain routing architecture. ACM Transactions on Networking, 2007. 